\title{
Distribuição de transtornos alimentares em indivíduos com diabetes melito do tipo 1 e do tipo 2: descrição de dois casos
}

\author{
Distribution of eating disorders in subjects with type 1 and type 2 diabetes mellitus: \\ a description of two cases
}

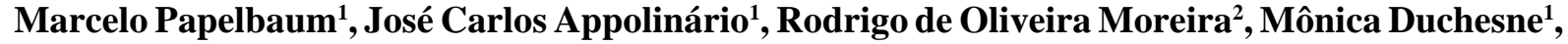 \\ Rosane Kupfer ${ }^{2}$, Walmir Coutinho ${ }^{2}$
}

${ }^{1}$ Grupo de Obesidade e Transtornos Alimentares, Instituto Estadual de Diabetes e Endocrinologia do Rio de Janeiro, Rio de Janeiro, RJ. Instituto de Psiquiatria, Universidade Federal do Rio de Janeiro (UFRJ), Rio de Janeiro, RJ. ${ }^{2}$ Grupo de Obesidade e Transtornos Alimentares, Instituto Estadual de Diabetes e Endocrinologia do Rio de Janeiro, Rio de Janeiro, RJ.

\begin{abstract}
Resumo
A presença de alterações do comportamento alimentar parece estar aumentada no diabetes melito (DM). Entretanto, a distribuição das diversas categorias de transtornos alimentares tende a se distinguir de acordo com a fisiopatologia do diabetes. O objetivo dessa apresentação é discutir dois casos distintos de ocorrência de transtornos alimentares no DM do tipo 1 (DM1) e no DM do tipo 2 (DM2). A paciente A é do sexo feminino, tem 19 anos e apresenta DM1 desde os 13 anos. Evidenciava sintomas depressivos proeminentes e, há 2 anos, passou a apresentar episódios de compulsão alimentar seguidos de vômitos auto-induzidos e omissão das doses de insulina com o objetivo de evitar ganho de peso. Em função desse comportamento, apresentou diversas internações associadas a uma piora do controle glicêmico. Após o uso de fluoxetina, houve remissão da psicopatologia alimentar e melhora do controle do DM. A paciente B possui 42 anos e é portadora do DM2 há 6 anos. Apresenta obesidade grau II e vinha exibindo, antes mesmo do diagnóstico do DM2, episódios de compulsão alimentar na ausência de comportamentos compensatórios, que prejudicavam o controle metabólico do diabetes. Foi iniciada fluoxetina até a dose de $60 \mathrm{mg} /$ dia, com remissão do descontrole alimentar, perda ponderal e redução da hemoglobina glicosilada. A incidência de transtornos alimentares no DM1 estaria associada com um aumento da preocupação com a forma corporal e a possibilidade da omissão do uso da insulina como comportamento compensatório. No DM2, a obesidade seria um dos fatores associados ao desenvolvimento da psicopatologia alimentar.
\end{abstract}

Descritores: Diabetes melito, transtorno alimentar, depressão.

\begin{abstract}
The presence of changes in eating behavior seems to be increased in diabetes mellitus (DM). However, the distribution of varied categories of eating disorders tends to be distinguished according to the physiopathology of diabetes. The objective of this report is to discuss two distinct cases of eating disorders in type 1 (T1DM) and type 2 diabetes mellitus (T2DM). Patient A is a 19-year-old female who has had T1DM since she was 13 years old. She presented prominent depressive symptoms and 2 years ago she started presenting binge eating episodes followed by self-induced vomits and insulin omission to avoid weight gain. Due to this behavior, she had several hospitalizations associated with worse glycemic control. After treatment with fluoxetine, there was remission of eating psychopathology and improvement in DM control. Patient B is a 42-year-old female who has had T2DM for 6 years. She has grade II obesity and had been showing, even before the diagnosis of T2DM, binge eating episodes in the absence of compensatory behaviors that jeopardized the metabolic control of DM. She started a treatment with fluoxetine up to $60 \mathrm{mg} /$ day, with remission of binge eating, weight loss and reduction in glycosylated hemoglobin. The incidence of eating disorders in T1DM seems to be associated with an increase in concern with body shape and the possibility of insulin omission as a compensatory behavior. In T2DM, obesity seems to be one of the factors associated with the development of eating psychopathology.
\end{abstract}

Keywords: Diabetes mellitus, eating disorder, depression.

Correspondência:

Marcelo Papelbaum, Rua Barão de Jaguaripe 63/401, Ipanema, CEP 22421-000, Rio de Janeiro, RJ. E-mail: mpapel@email.iis.com.br Copyright (C) Revista de Psiquiatria do Rio Grande do Sul - SPRS Recebido em 02/01/2007. Aceito em 21/03/2007. 


\section{Introdução}

O diabetes melito (DM) é uma doença crônica caracterizada por hiperglicemia persistente ${ }^{1}$. Apesar de todos os esforços no controle da doença, um grupo de pacientes com DM permanece com níveis glicêmicos elevados, mesmo seguindo orientações médicas específicas. A presença de uma comorbidade psiquiátrica tem sido uma das possibilidades aventadas quando um paciente com DM apresenta complicações no curso do seu tratamento.

Um grande conjunto de evidências sugere que haja um aumento de psicopatologia em pacientes com $\mathrm{DM}^{2-}$ ${ }^{4}$. A depressão e os transtornos alimentares (TA) encontram-se entre os transtornos psiquiátricos que mais despertam interesse nos clínicos e pesquisadores que lidam com pacientes com DM. Através de uma metanálise, Nielsen ${ }^{5}$ evidenciou um aumento na ocorrência de bulimia nervosa (BN) e de TA subclínicos entre mulheres portadoras do DM do tipo 1 (DM1) quando comparadas com indivíduos sem DM. Em um outro estudo, Jones et al. ${ }^{6}$ observaram um aumento nos episódios de compulsão alimentar (ECA) entre adolescentes com DM1, além de uma taxa de $11 \%$ de omissão das doses de insulina, com o objetivo de evitar ganho de peso. Com relação ao DM do tipo 2 (DM2), alguns estudos têm encontrado taxas elevadas do transtorno da compulsão alimentar periódica (TCAP) entre os indivíduos portadores de $\mathrm{DM}^{7,8}$.

Vários estudos têm investigado o impacto dos transtornos psiquiátricos no controle metabólico e na ocorrência de complicações clínicas associadas ao $\mathrm{DM}^{9,10}$. A presença da depressão parece estar associada a uma maior prevalência de complicações microvasculares entre os pacientes com $\mathrm{DM}^{10}$. Em relação aos TA, a presença de uma alteração do comportamento alimentar no DM1 tem se associado com um controle metabólico insatisfatório. No DM2, são escassos os estudos que avaliaram tal associação, mas, de uma forma geral, não foi evidenciado um impacto negativo dos TA no controle clínico da doença ${ }^{4}$.

O objetivo desta apresentação é discutir dois casos distintos de ocorrência de TA, um no DM1 e outro no DM2.

\section{Caso clínico 1}

A paciente Aé do sexo feminino, tem 19 anos e possui o diagnóstico de DM1 desde os 3 anos de idade. Refere períodos recorrentes de tristeza, desânimo e irritabilidade, com influência no funcionamento global e, sobretudo, no comportamento alimentar. Há 2 anos, passou a exibir, de forma mais freqüente (em uma média de duas a três vezes por semana), ECA, com a ingestão de grandes quantidades de doces em curto período de tempo, associada a uma sensação de descontrole. A paciente evidenciava grande preocupação com sua forma corporal e temia que, com o tratamento do DM, apresentasse um ganho ponderal. Dessa forma, para evitar ganho de peso, após os ECA, a paciente auto-induzia o vômito ou utilizava subdoses de insulina. A paciente já apresentou diversos episódios de cetoacidose diabética, muitos deles ocasionando internação hospitalar. Durante uma de suas internações hospitalares, iniciou-se fluoxetina até a dose de $40 \mathrm{mg} /$ dia, com acompanhamento psiquiátrico regular. A paciente apresentou remissão dos ECA e melhora do humor e não exibia mais comportamentos compensatórios. Houve uma diminuição das internações hospitalares, restringindo-se aos períodos em que a paciente não estava fazendo o uso adequado do antidepressivo.

\section{Caso clínico 2}

A paciente B é do sexo feminino, tem 41 anos e apresenta obesidade grau II e DM2 há 6 anos. Antes mesmo do diagnóstico do DM, a paciente exibia episódios freqüentes de descontrole alimentar, que, segundo a mesma, dificultavam o seguimento adequado das orientações dietéticas prescritas no tratamento da obesidade. Não foi evidenciada a prática de comportamentos compensatórios associados. Foi iniciada fluoxetina até a dose de $60 \mathrm{mg} / \mathrm{dia}$, com remissão dos ECA. A paciente passou a evidenciar uma perda ponderal importante e uma melhora do controle metabólico (Tabela 1). Como houve um aumento na ocorrência de episódios de hipoglicemia, a dose de insulina diária teve que ser reduzida.

Tabela 1 - Variáveis clínicas antes e após o uso de fluoxetina

\begin{tabular}{lcc}
\hline & $\begin{array}{r}\text { Antes do uso } \\
\text { de fluoxetina }\end{array}$ & $\begin{array}{c}\text { Em uso de } \\
\text { fluoxetina* }\end{array}$ \\
\hline Peso (kg) & 110,3 & 97,5 \\
$\begin{array}{l}\text { Hemoglobina glicosilada } \\
\text { (HbA1C) (\%) }\end{array}$ & 9 & 6 \\
\hline
\end{tabular}

*Os dados são referentes à avaliação após 1 ano do uso da fluoxetina.

\section{Discussão}

Os dois casos relatados exemplificam a ocorrência de alterações do comportamento alimentar em pacientes com o diagnóstico de DM. Como observado por 
Herpertz et al. ${ }^{7}$, a distribuição dos TA entre os dois pacientes descritos pareceu ocorrer de forma distinta de acordo com o tipo do DM. A paciente A já era portadora do DM1 na época em que se iniciaram os ECA. O aumento da preocupação com os hábitos alimentares e com o possível ganho de peso associado à terapia insulínica poderia ter predisposto a ocorrência de $\mathrm{BN}$ nessa paciente. Entretanto, o sexo feminino e a presença de uma valorização excessiva da forma corporal também são fatores de risco associados ao desenvolvimento dos TA. Dessa forma, a ocorrência casual da BN, sem relação direta com a presença do DM, é uma explicação possível. Em contrapartida, a paciente B já evidenciava ECA previamente ao diagnóstico do DM2. A necessidade de restrição alimentar imposta pelo tratamento do DM2 poderia ter acentuado a psicopatologia alimentar ${ }^{11}$. Em função das taxas elevadas de ECA entre indivíduos obesos (podendo chegar a $30 \%{ }^{12}$ ) e do fato de a paciente já exibir sintomas alimentares no momento do diagnóstico do DM, a ocorrência do TCAP na paciente B poderia estar diretamente associada à presença do excesso de peso. Dessa forma, como observado por Papelbaum et al. ${ }^{8}$, a BN parece ser o TA de ocorrência mais freqüente entre os indivíduos com DM1, e o TCAP, no DM2.

A presença de psicopatologia geral também é bastante freqüente entre os indivíduos com DM. Em cada três pacientes com DM, um apresenta depressão clínica (independente do tipo de DM) ${ }^{3}$. A paciente A evidenciava um transtorno depressivo recorrente, com piora dos sintomas alimentares durante os períodos de piora dos sintomas depressivos.

Ambas as pacientes alcançaram remissão dos sintomas alimentares através do tratamento farmacológico. O uso de inibidores seletivos da recaptação de serotonina (ISRS) se mostrou eficaz em reduzir os ECA e os sintomas depressivos ${ }^{13-15}$. Entretanto, não existem estudos que tenham investigado o uso desses medicamentos em amostras exclusivas de pacientes portadores de DM e de um TA associado. Alguns estudos avaliaram o uso de antidepressivos no tratamento da depressão em pacientes com $\mathrm{DM}^{3}$. A nortriptilina foi o primeiro antidepressivo a ser avaliado no tratamento da depressão nesses pacientes ${ }^{16}$. Apesar de acarretar uma melhora dos sintomas da depressão, ela apresentou um efeito hiperglicemiante direto. Por sua vez, os ISRS, como a sertralina e a fluoxetina, não parecem acarretar esse tipo de efeito metabólico. Além disso, os ISRS parecem aumentar a sensibilidade periférica à ação da insulina ${ }^{17}$.

Nos casos apresentados, a presença de um TA pareceu ter tido um impacto negativo no curso clínico do DM. No primeiro caso descrito, a paciente apresentava episódios freqüentes de cetoacidose diabética, em função da utilização inadequada da terapia insulínica. No segundo caso, a presença dos ECA poderia dificultar a adesão às orientações nutricionais específicas para o DM, ocasionando um ganho de peso associado à hiperfagia.

A presença de um TA tem se associado a um aumento de complicações clínicas do DM1. Nielsen ${ }^{5}$, através de uma metanálise, observou um risco aumentado de $33 \%$ de ocorrência de retinopatia em mulheres com DM1 e um TA, quando comparadas com indivíduos sem alteração do comportamento alimentar. Parece que, em função da categoria de TA, o impacto no curso clínico do DM1 pode ser diferente. Takki et al. ${ }^{18}$ evidenciaram um pior controle metabólico em pacientes com DM1 e BN do que em indivíduos com TCAP. A omissão do uso de insulina como método purgativo predominante entre os indivíduos com BN poderia justificar o maior prejuízo no controle metabólico do DM1. No DM2, entretanto, a presença de um TA não tem se associado com um aumento da ocorrência de complicações relacionadas ao DM. A ausência de comportamentos purgativos e o curso flutuante do TCAP poderiam explicar os achados negativos.

Em contrapartida, a melhora da psicopatologia alimentar através do uso da fluoxetina teve um impacto positivo no tratamento do DM dos casos clínicos descritos. A paciente com DM1 passou a aderir melhor ao esquema de insulina, diminuindo a freqüência de reinternações hospitalares. Por sua vez, a paciente com DM2 exibiu uma melhora objetiva do controle metabólico e perda ponderal significativa. Além disso, deve ser ressaltada a diminuição da dose total diária de insulina utilizada por essa paciente após o início do antidepressivo. A perda de peso associado ao tratamento e um possível aumento da sensibilidade periférica à ação da insulina pelo uso da fluoxetina poderiam justificar esse achado. Entretanto, deve ser levado em consideração que a remissão dos sintomas depressivos com o uso do ISRS também poderia justificar a melhora da evolução clínica do DM, através de um aumento na adesão às orientações médicas e nutricionais e um possível efeito metabólico direto ${ }^{19}$.

\section{Conclusões}

Os casos relatados exemplificam a comorbidade entre o DM e os TA. De uma forma geral, os TA parecem se apresentar de forma distinta no DM1 e no DM2. A BN tem sido observada como o TA de ocorrência predominante no DM1, e o TCAP, no DM2. Além disso, a omissão das doses de insulina parece ser o método compensatório predominante entre as pacientes com DM1 que exibem uma alteração do comportamento alimentar. Apesar de os TA poderem 
ocasionar uma piora do controle clínico do DM1, o tratamento dos sintomas alimentares poderia acarretar uma melhora do controle metabólico dos pacientes com DM1 e DM2. São necessários estudos que avaliem o tratamento dos TA em amostras exclusivas de pacientes com DM.

\section{Referências}

1. Alberti KG, Zimmet PZ. Definition, diagnosis and classification of diabetes mellitus and its complications. Part 1: diagnosis and classification of diabetes mellitus, provisional report of a WHO consultation. Diabetic Med. 1998;15(7):539-53.

2. Papelbaum M, Moreira RO, Coutinho W, Ellinger VCM, Sichieri R, Coutinho E, et al. Diabetes mellitus e transtornos alimentares: uma revisão sistemática. J Bras Psiquiatr. 2004;53(3):163-73.

3. Moreira RO, Papelbaum M, Appolinario JC, Matos AG, Coutinho WF, Meirelles RMR, et al. Diabetes mellitus e depressão: uma revisão sistemática. Arq Bras Endocrinol Metab. 2003;47(1):19-29.

4. Crow S, Kendall D, Praus B, Thuras P. Binge eating and other psychopathology in patients with type II diabetes mellitus. Int J Eat Disord. 2001;30(2):222-6.

5. Nielsen S. Eating disorders in females with type 1 diabetes: an update of a meta-analysis. Eur Eat Disord Rev. 2002;10(4):241-54.

6. Jones JM, Lawson ML, Daneman D, Olmsted MP, Rodin G. Eating disorders in adolescent females with and without type 1 diabetes: cross sectional study. BMJ. 2000;320(7249):1563-6.

7. Herpertz S, Wagener R, Albus C, Kocnar M, Wagner R, Best F, et al. Diabetes mellitus and eating disorders: a multicenter study on the comorbidity of the two diseases. J Psychosom Res. 1998;44(34):503-15.

8. Papelbaum M, Appolinário JC, Moreira RO, Ellinger VC, Kupfer R, Coutinho WF. Prevalência de transtornos alimentares e comorbidade psiquiátrica em uma amostra clínica de pacientes com diabetes mellitus do tipo 2. Rev Bras Psiquiatr. 2005;27(2):135-8.

9. Herpertz S, Albus C, Wagener R, Kocnar M, Wagner R, Henning A, et al. Comorbidity of diabetes and eating disorders. Does diabetes control reflect disturbed eating behavior? Diabetes Care. 1998;21(7):1110-6.

10. Rydall AC, Rodin GM, Olmsted MP, Devenyi RG, Daneman D. Disordered eating behavior and microvascular complications in young women with insulin-dependent diabetes mellitus. N Engl J Med. 1997;336(26):1849-54.

11. Yanovski SZ. The chicken or the egg: binge eating disorder and dietary restraint. Appetite. 1995;24(3):258.

12. de Zwaan M. Binge eating disorder and obesity. Int J Obes Relat Metab Disord. 2001;25 Suppl 1:S51-5.

13. Goodnick PJ, Kumar A, Henry JH, Buki VM, Goldberg RB. Sertraline in coexisting major depression and diabetes mellitus. Psychopharmacol Bull. 1997;33(2):261-4.

14. Lustman PJ, Freedland KE, Griffith LS, Clouse RE. Fluoxetine for depression in diabetes: a randomized double-blind placebo controlled trial. Diabetes Care. 2000;23(5):618-23.

15. Appolinario JC, McElroy SL. Pharmacological approaches in the treatment of binge eating disorder. Curr Drug Targets. 2004;5(3):301-7.

16. Lustman PJ, Griffith LS, Clouse RE, Freedland KE, Eisen SA, Rubin $\mathrm{EH}$, et al. Effects of nortriptyline on depression and glycemic control in diabetes: results of a double-blind, placebo controlled trial. Psychosom Med. 1997;59(3):241-50.

17. McIntyre RS, Soczynska JK, Konarski JZ, Kennedy SH. The effect of antidepressants on glucose homeostasis and insulin sensitivity: synthesis and mechanisms. Expert Opin Drug Saf. 2006;5(1):157-68.

18. Takii M, Komaki G, Uchigata Y, Maeda M, Omori Y, Kubo C. Differences between bulimia nervosa and binge-eating disorder in females with type 1 diabetes: the important role of insulin omission. $J$ Psychosom Res. 1999;47(3):221-31.

19. Lustman PJ, Clouse RE, Nix BD, Freedland KE, Rubin EH, McGill $\mathrm{JB}$, et al. Sertraline for prevention of depression recurrence in diabetes mellitus: a randomized, double-blind, placebo-controlled trial. Arch Gen Psychiatry. 2006;63(5):521-9. 